\title{
Obtaining a Clearer Picture through Format Upgrades
}

\section{By Becky Smith}

Determining the longevity of material formats has become an increasing challenge in today's technological landscape. When it comes to audiovisual resources, libraries face the gamut from VHS to LaserDiscs to DVDs to Blu-ray. This article describes my recent efforts at Memorial University of Newfoundland's Music Resource Centre to update its collection of audiovisual materials. In particular, I will be looking at the issues involved in updating such a collection and my success rate for replacement.

\section{Background}

Memorial University's libraries consist of the main Queen Elizabeth II Library, four branches, and two resource or material centres, of which the Music Resource Centre (MRC) is one. Due to space constraints, the MRC's collection consists primarily of music scores, sound recordings and audiovisual material. Prior to 2011, the music collections were maintained on a part-time basis by a variety of librarians, so retrospective maintenance activities were rarely undertaken. Upon my arrival in July 2011, the audiovisual collection contained a mixture of DVDs, LaserDiscs and VHS cassettes.

In the fall of 2011, I was given additional funding to update the MRC collections, with a deadline of December 2011 to spend the funds. Since there was not time to thoroughly evaluate the collection, I consulted with the Music faculty to identify areas which could be improved. Based in part on these consultations, I initially decided to focus on upgrading the LaserDisc collection to DVDs as one of the improvements to the collection. Shortly into the project, I realized the VHS cassettes should also be replaced, since a number of these items were showing signs of deterioration. As well, I consider the replacement of these items by DVDs important due to student unfamiliarity with the formats and how to use them.

\section{Procedure}

The goal for replacement was to find exact copies of specific performances, so that only format, rather than content, would change. The short time frame, in combination with time constraints

Becky Smith is the Music Librarian at Memorial University Libraries. 
imposed by my regular duties, did not allow for a detailed analysis of the collection to determine if certain works were no longer needed or desired. As I, at this point, did not know the whole history of the collection, I made the assumption that all the performances were added to fill particular needs. Another reason for replacing the content of the collection, rather than strategic replacement, was the lack of usage statistics. At the time of this project, circulation of MRC items had only been automated for three or four years. A final reason for this method was concern over copyright. While, in many cases, I could obtain an exact copy of the performance through in-house copying, I was uncertain of the legality of such preservation copies, and decided to err on the side of caution and purchase new copies.

The first step for replacing the LaserDiscs and VHS cassettes was the creation of a complete bibliographic listing of the titles from our catalogue, including production and performer details. This list was first used to determine if a DVD version was already in the collection, as some titles had been previously updated. I was also able to determine the availability of performances through our online streaming products. If a performance was not already available to our patrons, the internet was then scoured to find vendors for DVD replacements. When no vendor was found, I assumed that either the performance was not transferred to DVD, or that the DVD was no longer in production. The availability of a given title on DVD was the primary consideration for its replacement. For items no longer in production, second-hand titles were purchased if the status as an exact copy could be determined. Since second-hand vendors often provide limited information, these purchases were rare.

Another consideration was space. For example, for works with multiple performances on LaserDisc and/or VHS, I tried to replace the most valuable items based on aspects such as performers, orchestras or labels.

\section{LaserDisc Results}

The LaserDisc collection in the MRC consisted of 130 individually call-numbered items. Of these, 18 (or approximately 14\%) were already in the collection in DVD format and could be removed. For 11 items (8\%), either an exact copy of the work was available through an online streaming product or there were alternate performances of the same work already on DVD or through streaming. Seventeen items (14\%) could not be found on DVD and thus could not be replaced. Fifteen further items (12\%) have proven troublesome. Nine of these presented issues such as repackaging or re-titling, but in the end, DVDs were purchased that appeared to fill the content need, though their status as exact copies required more scrutiny upon arrival. The second group consisted of six items that appeared to be exact copies and should not have been 
problematic to obtain. As they have not arrived, for unknown reasons, I am unable to determine if the performance is actually available.

By the end of this process, 68 (52\%) of LaserDiscs were replaced by DVDs. Adding these to the $14 \%$ already replaced, and the $8 \%$ available online or in alternate performances, $74 \%$ of the LaserDisc collection has been successfully replaced.

\section{Figure 1: LaserDisc Replacement}

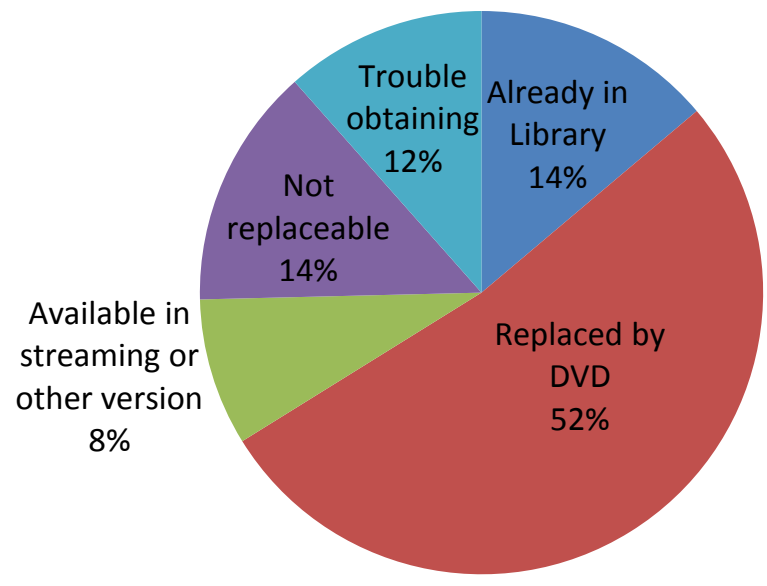

The LaserDisc collection included a couple of surprising items probably not suitable for the research needs of the School of Music, but interesting nonetheless. The first was an animated version of The Magic Flute, intended for children, with Tamino voiced by Mark Hamill. While this work might pique the interest of those who know and love the original Star Wars trilogy, it is not likely to suit current research needs. While it won't be replaced by DVD, it will not be immediately deselected. The second example was the film Allegro Non Troppo. This (also) animated work by Italian Bruno Bozzetto could be compared to Disney's Fantasia, as it uses the music of Debussy, Stravinsky, Vivaldi and others as the connective tissue for animations. While viewing portions of this work, I could observe the different perspectives used by Bozzetto compared with Disney to create a, perhaps, more mature animation. Like the animated Magic Flute, Allegro Non Troppo will be kept on LaserDisc for now.

The most disappointing search was for a replacement of the LaserDisc The Complete Show Boat. This item contains three full length productions of Show Boat from 1929, 1936 and 1951, with the Show Boat sequence from Till The Clouds Roll from 1946. Faculty identified the 1936 version, with the iconic Paul Robeson, as the most desirable performance, but only the 1951 version was available on DVD. On the other end of the spectrum was the search for Wagner's 
Der Ring des Nibelungen conducted by Daniel Barenboim and issued by Teldec. While searching for a DVD of this work, I found many traces through vendors who listed it as unavailable or out of stock but without sufficient production information to confirm the performance. I initially concluded the item had been available and was now out of print, perhaps never to be released again. Something about the results of my searches made me persist, however, and in the end, I was pleasantly surprised to find this item would soon be available on DVD from Kultur.

\section{VHS Results}

The process of replacing the VHS cassettes was initially easier than the LaserDiscs, since more items had already been replaced by DVDs. Out of approximately 117 items (depending on whether you count call numbers or individual cassettes), 27 (23\%) had been replaced by the start of the project. The search for replacements, conducted in the same way as for LaserDiscs, resulted in 38 items (32\%) successfully ordered and received. As with the LaserDiscs, some replacements have been ordered but not received, and the source of the trouble has not been identified. By the end of the project, I determined that 37 items (32\%) were not replaceable. Of these, the two most desirable items were the PBS production Dancing, and the series Man and Music. As with the LaserDiscs, between the items already replaced and the new purchases, more than half the collection has been updated. It is, however, interesting to note the difference in the amount deemed irreplaceable: $14 \%$ of LaserDiscs versus $32 \%$ of VHS. Whether these numbers reflect unique content in the library's collection, or suggest that transfers to DVD were more common for LaserDiscs than VHS, is beyond the scope of this report.

\section{Figure 2: VHS Replacement}

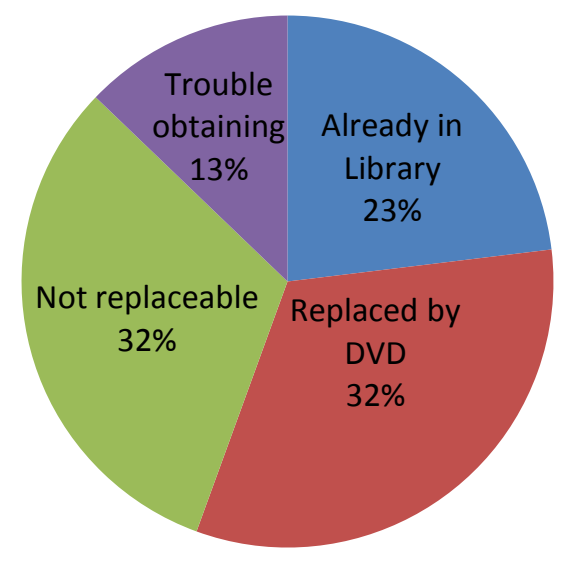




\section{Conclusion}

Having the time and funding available to replace outdated formats does not happen every day. My experience suggests that this project was a worthwhile effort and increased the usability of the collection. While not all items could be replaced, many additional works are now accessible to students who may have been hesitant to use unfamiliar formats and equipment. The instructors now have greater variety in content for their classrooms, without the need for additional equipment or the worry that an item may no longer play. In a perfect world, a more targeted approach would have been undertaken, but this process at least ensured no important works were lost. One can only hope that valuable items not currently replaceable will one day be made available in a more accessible format. What that format will be is anyone's guess. 